\title{
Imagen que poseen los españoles de China como destino turístico
}

\author{
China as a tourist destination: how Spaniards see it
}

\author{
José Ramón Cardona (RAMÓN CARDONA, J.)
}

\begin{abstract}
RESUMEN - China no sólo se está convirtiendo en un importante destino turístico internacional, sino que también tiene un gran potencial como mercado emisor. España, por su parte, se ha posicionado entre los tres principales destinos turísticos del mundo y, como mercado emisor, está creciendo gradualmente en importancia. Hay un gran potencial de incremento de los flujos turísticos entre España y China en ambas direcciones. Este trabajo analiza la imagen de los españoles sobre China como país y como destino turístico y su familiaridad con la realidad de este país asiático en términos de turismo. Para ello, se realizaron 1.063 entrevistas en Mallorca, con preguntas cerradas. Los resultados apuntan a una importante intención potencial de visitar China, aunque también demuestra la escasa familiaridad con la realidad de China como destino turístico. China es vista como un destino de turismo cultural y urbano, principalmente. Los resultados también parecen indicar la existencia de grandes inhibidores (coste del viaje, daño medioambiental, distancia entre China y España, entre otros).
\end{abstract}

Palabras clave: España; China; Percepciones; Conocimientos; Imagen del destino turístico.

ABSTRACT - China is not only becoming a major international tourist destination, it also has huge potential as an issuing market. Spain, meanwhile, has long ranked among the world's top three tourist destinations and, as an issuing market it is gradually growing in importance. There is high potential for an increase in tourist flows between Spain and China in both directions. This paper analyzes the image of Spanish people on China as a country and as a tourist destination and their familiarity with the reality of this Asiatic country in terms of tourism. For this, 1,063 interviews were conducted in Mallorca, with closed questions. The results suggest a potential significant intention to visit China, although it also reveals very little familiarity with the reality of China as a tourist destination. China is seen as a destination for cultural and urban tourism, mainly. The results also seem to indicate the existence of big inhibitors (travel cost, environmental damage, distance between China and Spain, among others).

Key words: Spain; China; Perceptions; Knowledge; Tourism destination image.

\footnotetext{
* Formación: Licenciado en Administración y Dirección de Empresas (Universitat de les Illes Balears), Licenciado en Economía (Universitat de les Illes Balears) y Doctorado en Economía de la Empresa (Universitat de les Illes Balears). Investigador colaborador del grupo de Dirección y Gestión de Empresas y Destinos Turísticos (Universitat de les Illes Balears). Dirección: Casa Can Pilot (Buscastell), n ${ }^{\circ}$ 15.047. C.P. 07820 - Sant Antoni de Portmany (Illes Balears) - España. Teléfono: (+34) 971343789 / 6968085 32. E-mail: jramon.cardona@terra.com
} 


\section{INTRODUCIÓN}

La imagen o percepción de un destino turístico (BIGNÉ; SÁNCHEZ; SÁNCHEZ, 2001; CHEN, 2005; CROMPTON, 1979; KIM; MORRISON, 2005; LEE, 1997; LEE; LEE; LEE, 2005; NOH, 2007; TASCI; GARTNER; CAVUSGIL, 2007) es una de las áreas más estudiadas en la literatura (STEPCHENKOVA; MORRISON, 2008). En la investigación turística, la definición de imagen "incluye las percepciones o las impresiones que tiene una persona de un lugar" (MCCLINCHEY, 1999, p. 9). Aunque los estudiosos del turismo han propuesto numerosas definiciones (LI; VOGELSONG, 2006), la mayoría tienden a estar de acuerdo en que la imagen es la impresión general de un lugar (LI et al., 2009). Los estudios de imagen de los destinos turísticos son importantes (HUNT, 1975) y sirven para: entender el conocimiento del cliente y el conocimiento de marca de un destino; formular el desarrollo de productos y estrategias de posicionamiento de destino, y evaluar la eficacia de los esfuerzos de promoción del destino y predecir la intención de conducta de los turistas (PAN; LI, 2011).

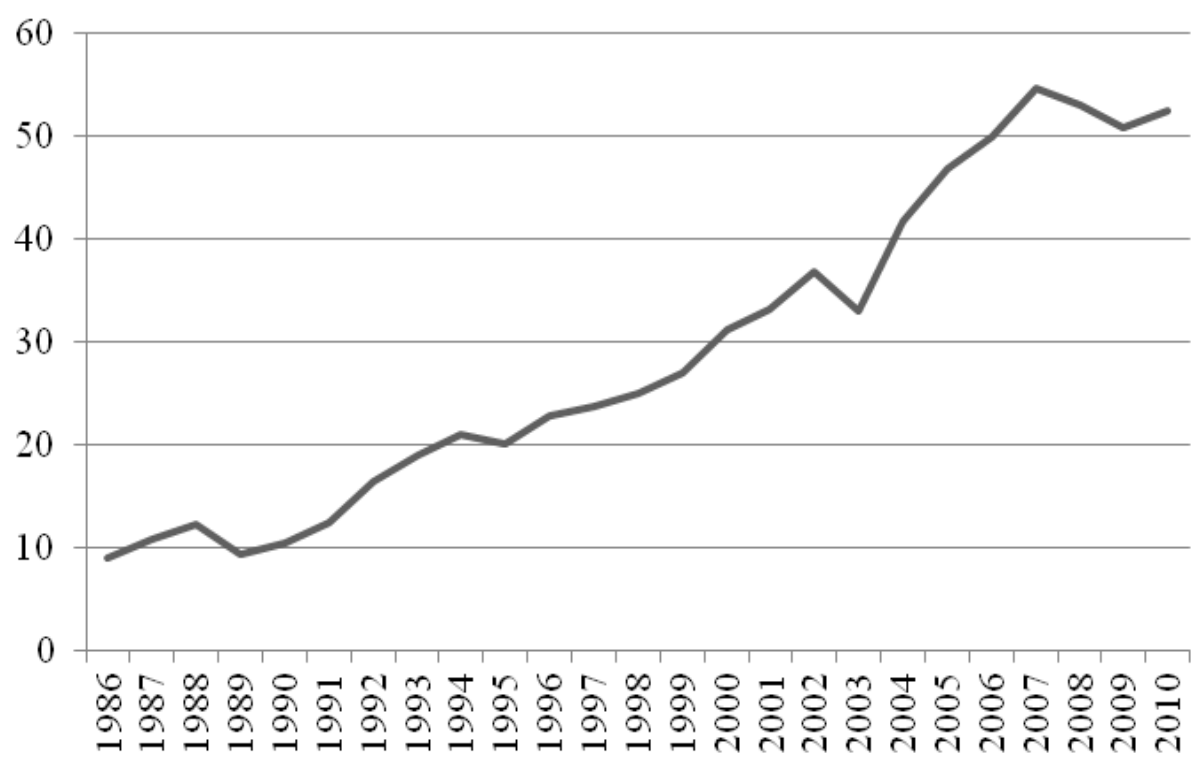

FIGURA 1 - LLEGADA DE TURISTAS A LA CHINA CONTINENTAL (MILLONES DE TURISTAS).

Fuente: National Tourism Administration of China y World Tourism Organization (año 2012).

China se está convirtiendo en un destino turístico de primera magnitud a nivel global. Desde 1978 se ha producido un aumento constante en la llegada de turistas 
(Figura 1), aunque mayoritariamente llegan de Hong Kong, Macao y Taiwán (Cuadro 1), y en la publicación de investigaciones sobre turismo en China (TSANG; HSU, 2011). El principal atractivo turístico chino es el patrimonio cultural (LI, 2008; YANG; LIN; HAN, 2010). Las previsiones de la Organización Mundial de Turismo (UNWTO, 2011) sitúan a China en el primer lugar, a nivel mundial, como destino receptor de llegadas turísticas internacionales en 2020. Al mismo tiempo, las altas tasas de crecimiento económico de China durante la última década, unidas al gran tamaño de su población, hacen que China sea vista como un importante mercado turístico emisor en el futuro (UNWTO, 2011).

CUADRO 1 - LLEGADAS DE TURISTAS A LA CHINA CONTINENTAL.

\begin{tabular}{|l|r|r|}
\cline { 2 - 3 } \multicolumn{1}{c|}{} & $\mathbf{2 0 0 6}$ & \multicolumn{1}{c|}{$\mathbf{2 0 0 7}$} \\
\hline Llegada de visitantes & $\mathbf{1 2 4 . 9 4 2 . 2 0 0}$ & $\mathbf{1 3 1 . 8 7 3 . 3 0 0}$ \\
Extranjeros. & 22.210 .300 & 26.109 .700 \\
Hong Kong. & 73.909 .700 & 77.948 .900 \\
Macao. & 24.408 .700 & 23.186 .800 \\
Taiwán. & 4.413 .500 & 4.627 .900 \\
\hline Llegadas de turistas (pernoctan). & $\mathbf{4 9 . 9 1 3 . 4 0 0}$ & $\mathbf{5 4 . 7 1 9 . 8 0 0}$ \\
Extranjeros. & 18.106 .100 & 21.398 .900 \\
Hong Kong y Macao. & 27.966 .800 & 29.299 .700 \\
Taiwán. & 3.840 .500 & 4.021 .200 \\
\hline
\end{tabular}

Fuente: National Tourism Administration of the people's republic of China (año 2012).

España ha sido, históricamente, un destino turístico de primera magnitud, figurando, sistemáticamente, entre los tres primeros destinos a nivel global durante las últimas décadas (UNWTO, 2011). Pero, además, en los años recientes, España está incrementando progresivamente su importancia como mercado turístico emisor (datos del INE). Una evolución que va paralela a su creciente nivel de desarrollo económico y al progresivo incremento de renta disponible entre sus habitantes, aunque desde 2009 se ha producido un cierto descenso debido a la crisis (datos del INE).

Las escasas estadísticas oficiales existentes muestran unos números de visitantes en ambos sentidos relativamente escasos. Así, los visitantes españoles a China estarían situados en torno a los 114.500 en el 2009 según la China National Tourist Office (Cuadro 2), mientras que las cifras de turistas chinos hacia España son, incluso, menores: unos 67.500 en el 2008 según el Instituto de Turismo de España (2009). 
CUADRO 2 - ESPAÑOLES QUE HAN VISITADO CHINA.

\begin{tabular}{|l|r|r|r|r|r|}
\multicolumn{1}{c|}{} & $\begin{array}{c}\text { Trabajo / } \\
\text { Estudios }\end{array}$ & Ocio / Turismo & $\begin{array}{c}\text { Visitar amigos } \\
\text { o familiares }\end{array}$ & \multicolumn{1}{c|}{ Otros } & \multicolumn{1}{c|}{ Total } \\
\hline $\mathbf{2 0 0 6}$ & 35.997 & 84.354 & 320 & 8.581 & 129.252 \\
$\mathbf{2 0 0 7}$ & 49.280 & 80.262 & 57 & 7.132 & 136.731 \\
$\mathbf{2 0 0 8}$ & 33.967 & 68.156 & 61 & 10.040 & 112.224 \\
$\mathbf{2 0 0 9}$ & 30.400 & 71.300 & 100 & 12.800 & 114.600 \\
\hline
\end{tabular}

Fuente: China National Tourist Office (CNTO, año 2012)

Existe, por tanto, un gran potencial para el incremento de los flujos turísticos entre ambos países y en ambos sentidos. Sin embargo, revisando la literatura académica existente, se constata la práctica inexistencia de estudios encaminados a incrementar el grado de conocimiento de ambos mercados y su potencial emisor recíproco. Diversos estudios previos han analizado la imagen que tienen en la China continental de otros destinos turísticos (GUO; KIM; TIMOTHY, 2007; VAN DER VEEN; SONG, 2010), y en especial de Australia (LEE; LOCKSHIN, 2011; SPARKS; PAN, 2009), Hong Kong (HUANG; HSU, 2009; LAW; CHEUNG, 2010; LEUNG; LAW; LEE, 2011; YANG; HE; GU, 2012), Macao (MCCARTNEY; BUTLER; BENNETT, 2008), Japón (KIM; GUO; AGRUSA, 2005; YANG; HE; GU, 2012), Singapur, Tailandia, Corea del Sur, Egipto y Alemania (KIM; GUO; AGRUSA, 2005). Algunos estudios analizan la imagen de China en Norteamérica (GIBSON; QI; ZHANG, 2008; GREEN et al., 2010; HESLOP; NADEAU; O’REILLY, 2010; LI et al., 2009; PAN; LI, 2011; SHANI et al., 2010). Los estudios realizados sobre los mercados turísticos emisores hacia China, contemplan Europa como un todo y, en el caso de particularizar, contemplan tan sólo determinados mercados emisores europeos (Reino Unido, Francia, Alemania e Italia), ignorando, sistemáticamente, a España como mercado emisor (JEFFREY; XIE, 1995; YANG; LIN; HAN, 2010). Tan sólo Seguí y Capellà (2006) estudian determinados aspectos del mercado turístico español hacia China. Por otra parte, los artículos centrados en el análisis de la imagen o la percepción que los visitantes tienen de China (CHOI; CHAN; WU, 1999; SU; WALL, 2009; ZHANG; CHOW, 2004), se centran en ubicaciones muy concretas o en productos turísticos muy específicos, por ejemplo los Juegos Olímpicos de Beijing (GREEN et al., 2010; HESLOP; NADEAU; O’REILLY, 2010). Pero, en ningún caso, intentando valorar la percepción o imagen global de China como país y/o como destino turístico en su conjunto. Además, los análisis se basan en las opiniones de visitantes y no analizan dichas cuestiones entre el mercado potencial. 
La novedad de este estudio radica en que, por primera vez, se contempla España como mercado emisor hacia China, se intenta determinar la imagen de China, como país y destino turístico, entre la población española y se realiza un estudio basado en el conjunto del mercado potencial y no solamente sobre aquellos que han visitado China.

\section{METODOLOGIA}

Los estudios sobre la imagen de destinos turísticos han utilizado enfoques estructurados o no estructurados (BALOGLU; MANGALOGLU, 2001; ECHTNER; RITCHIE, 1993; PAN; LI, 2011; SELBY; MORGAN, 1996), pero el estructurado es utilizado mucho más ampliamente que el no estructurado (BIGNÉ; SÁNCHEZ; SANZ, 2009; PIKE, 2002). Algunos investigadores han intentado combinar enfoques estructurados y no estructurados para capturar mejor los diferentes componentes de la imagen (BALOGLU; MANGALOGLU, 2001; CHOI; CHAN; WU, 1999; ECHTNER; RITCHIE, 1993; SELBY; MORGAN, 1996).

La metodología seguida en este artículo es el enfoque estructurado, compartiendo similitudes con otros estudios (CHOI; TKACHENKO; SIL, 2011; SPARKS; PAN, 2009; STEPCHENKOVA; MORRISON, 2008). El enfoque estructurado evalúa la imagen cognitiva y afectiva mediante una lista estructurada de múltiples atributos (BALOGLU; MANGALOGLU, 2001). Por lo general, los investigadores han agrupado estos atributos en varias dimensiones de análisis factorial (GALLARZA; SAURA; GARCÍA, 2002). Los enfoques estructurados gozan de ventajas tales como la idoneidad para la codificación y la facilidad de análisis (SELBY; MORGAN, 1996).

Para la realización del estudio se ha recurrido a la realización de entrevistas en la isla de Mallorca. La elección de Mallorca para el trabajo de campo se debe a la conveniencia y disponibilidad de los investigadores para la realización del trabajo de campo, mediante entrevistas personales. Cabe mencionar que una pequeña muestra realizada en la provincia de Sevilla mostraba resultados similares, haciendo plausible considerar la muestra de Mallorca una buena aproximación al total de España. La encuesta está estructurada en diversas partes. Una primera parte compuesta por 22 ítems 
consistentes en afirmaciones referentes a China en general y como destino turístico con escala Likert de 5 puntos, de "muy en desacuerdo" a "muy de acuerdo", y una sexta opción de "no sabe/no contesta". Una segunda parte compuesta por 16 ítems que permiten valorar diversos elementos de la oferta turística china, también mediante una escala Likert. Los ítems de estos dos apartados se elaboraron a partir de trabajos teóricos (ECHTNER; RITCHIE, 1991; JENKINS, 1999) o prácticos que analizaban destinos concretos (CHOI; CHAN; WU, 1999; JEFFREY; XIE, 1995; SU; WALL, 2009; ZHANG; CHOW, 2004), aunque peculiaridades como el hecho de estudiar la imagen de China en general entre un mercado potencial, y no entre visitantes de un lugar concreto, ha obligado a la creación de una escala propia que contiene elementos que resultan de importancia en estudios como el de Sparks y Pan (2009) o en el de Yang, Lin y Han (2010). La última parte estaba compuesta por 8 preguntas cerradas referentes a características socio-demográficas del entrevistado y a la visita o intención de visita a China (Cuadro 3).

CUADRO 3 - VARIABLES DEMOGRÁFICAS DE LA MUESTRA.

\begin{tabular}{|l|r|r|}
\hline Características & Frecuencia & \multicolumn{1}{|c|}{$\%$} \\
\hline Sexo (n=1050) & & \\
Hombre. & 472 & $45.0 \%$ \\
Mujer. & 578 & $55.0 \%$ \\
\hline Edad (n=1061) & & \\
Menos de 25. & 426 & $40.2 \%$ \\
$25-34$ & 213 & $20.1 \%$ \\
$35-44$ & 114 & $10.7 \%$ \\
$45-54$ & 191 & $18.0 \%$ \\
$55-64$ & 77 & $7.3 \%$ \\
65 o más. & 40 & $3.8 \%$ \\
\hline Nivel de Estudios (n=1027) & & \\
Sin Estudios. & 28 & $2.7 \%$ \\
Estudios Primarios. & 137 & $13.3 \%$ \\
Estudios Secundarios. & 384 & $37.4 \%$ \\
Estudios Universitarios. & 478 & $46.5 \%$ \\
\hline Ocupación principal (n=1056) & & \\
Asalariado (Empleado). & 290 & $27.5 \%$ \\
Asalariado (Mando Intermedio). & 58 & $5.5 \%$ \\
Asalariado (Alto Ejecutivo). & 15 & $1.4 \%$ \\
Trabaja para la Administración. & 77 & $7.3 \%$ \\
Empresario (Propietario Empresa). & 59 & $5.6 \%$ \\
Profesional Liberal. & 31 & $2.9 \%$ \\
Labores de la Casa. & 45 & $4.3 \%$ \\
Estudiante. & 377 & $35.7 \%$ \\
Retirado / Jubilado. & 50 & $4.7 \%$ \\
Sin Empleo. & 52 & $4.9 \%$ \\
Otros. & 2 & $0.2 \%$ \\
\hline
\end{tabular}


Continuación...

\begin{tabular}{|l|r|r|}
\hline Ingresos netos familiares anuales $(\mathbf{n = 9 7 2})$ & 220 & $22.6 \%$ \\
Menos de $15.000 €$ & 386 & $39.7 \%$ \\
De $15.001 €$ a $30.000 €$ & 206 & $21.2 \%$ \\
De $30.001 €$ a $45.000 €$ & 92 & $9.5 \%$ \\
De $45.001 €$ a $60.000 €$ & 40 & $4.1 \%$ \\
De $60.001 €$ a $75.000 €$ & 28 & $2.9 \%$ \\
Más de $75.000 €$ & & \\
\hline Visitado China $(\mathbf{n = 1 0 6 1 )}$ & 38 & $3.6 \%$ \\
Si & 1023 & $96.4 \%$ \\
No & & \\
\hline
\end{tabular}

Fuente: elaboración propia.

Las entrevistas se realizaron durante el primer semestre de 2011. Finalmente se obtuvieron 1.063 entrevistas validas que fueron procesadas mediante el paquete estadístico DYANE Versión 4 (SANTESMASES, 2009). Se procedió a la realización del cálculo de las puntuaciones medias y a comprobar, mediante prueba t de media de una muestra, si pueden considerarse distintas de 3, es decir "Indiferente".

\section{RESULTADOS}

China era vista por los españoles como un país con un gran patrimonio histórico y cultural $(84,2 \%)$ que, sin duda, va a convertirse en la gran potencia del futuro $(81,1 \%)$, tanto a nivel económico como político. En términos económicos, para la mayoría de entrevistados $(66,9 \%)$ China ya podía considerarse un país desarrollado, aunque un significativo porcentaje $(24,1 \%)$ manifestaba estar en desacuerdo con tal afirmación. El elevado nivel de contaminación era un componente claramente negativo de la imagen del país: tres de cada cuatro entrevistados tenían la percepción que el rápido proceso de desarrollo económico chino ha provocado un gran impacto negativo sobre el medio ambiente del país.

La inmensa mayoría de los entrevistados $(96,4 \%)$ no habían visitado China, unos resultados que no sorprenden dado el escaso número de visitantes españoles a China que muestran las estadísticas oficiales disponibles. La realidad, por tanto, es que los números son exiguos, pero lo interesante es el potencial de futuro que parece desprenderse de las respuestas dadas. Así, el 36,3\% manifestaban que tenían previsto visitar China en los próximos años, en su práctica totalidad por motivos de ocio y turismo. Entre los que manifestaban la intención de visitar el país, más de la mitad 
tenían estudios universitarios. Alternativamente, y con una consideración más a largo plazo y condicionada a que las circunstancias lo permitan, el 54\% manifestaba estar de acuerdo en que 'China es un país realmente atractivo que les gustaría algún día visitar'. El potencial emisor de España hacia China parece, pues, significativo.

\subsection{GRADO DE CONOCIMIENTO DEL DESTINO}

Esta elevada intención de visitar China contrastaba con el relativo desconocimiento de los atractivos turísticos concretos de China como destino turístico (Cuadro 4). Mientras queda patente que la mayoría de los entrevistados tenían claro que China es un país con muchos sitios interesantes para visitar y que uno de sus mayores atractivos radica en su cultura y sus diferencias con Occidente, tan sólo el 57\% estaban de acuerdo en que es un destino turístico muy importante a nivel mundial (el 25,5\% no sabía y el $17,1 \%$ se manifestaba en desacuerdo). Tan sólo el $26,3 \%$ pensaba que la oferta china es amplia y variada. En los aspectos que hacen referencia a atributos importantes de un destinos turístico, tales como la amplitud y variedad de la oferta turística, su nivel de calidad, la seguridad del destino, las infraestructuras de transportes y comunicaciones, el conocimiento de idiomas extranjeros por parte de la población y la hospitalidad de los residentes, el aspecto más llamativo era el elevado porcentaje de entrevistados que manifestaban que no lo sabían y, por tanto, no tenían una postura clara de acuerdo o desacuerdo. Tan sólo en lo que hace referencia a la modernidad de las infraestructuras de transportes y comunicaciones se obtenían porcentajes relativamente elevados de acuerdo $(42,9 \%)$. El resto de componentes o atributos del destino (que con puntuaciones altas implican, ceteris paribus, mayor atractivo del destino) no superaban el $30 \%$ de grado de acuerdo. Más que los porcentajes de desacuerdo, llaman la atención los elevados porcentajes de entrevistados que no tenían una postura clara al respecto, lo que parece sugerir un elevado grado de desconocimiento sobre la realidad del destino. Un aspecto que refuerza el hecho de que tan sólo el 19,2\% de los entrevistados pensaran que se dispone de mucha y buena información sobre la oferta turística existente en China. Lo que sí parecen tener más claro los entrevistados es que al viajar a China es mejor hacerlo mediante la 
contratación de un paquete turístico, puesto que viajar 'por libre' dentro de China no resulta fácil para un extranjero.

\section{CUADRO 4 - VALORACIÓN GENERAL DE CHINA}

\begin{tabular}{|c|c|c|c|c|}
\hline & 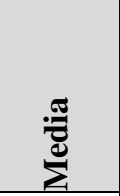 & 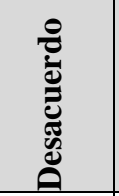 & 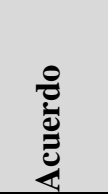 & 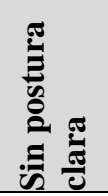 \\
\hline En China hay muchos sitios muy interesantes para visitar. & $4,14 *$ & $5,1 \%$ & $79,3 \%$ & $15,6 \%$ \\
\hline $\begin{array}{l}\text { Uno de los mayores atractivos de China es su cultura y sus } \\
\text { diferencias con occidente. }\end{array}$ & $4,23 *$ & $4,3 \%$ & $84,6 \%$ & $11,1 \%$ \\
\hline China es un destino turístico muy importante a nivel mundial. & $3,59 *$ & $17,1 \%$ & $57,4 \%$ & $25,5 \%$ \\
\hline La oferta turística china es amplia y variada. & 3,06 & $26,3 \%$ & $28,1 \%$ & $45,5 \%$ \\
\hline China dispone de una oferta turística de calidad internacional. & $3,25 *$ & $15,1 \%$ & $27,4 \%$ & $57,5 \%$ \\
\hline China es un lugar seguro para los turistas. & 2,97 & $29,9 \%$ & $28,0 \%$ & $42,1 \%$ \\
\hline $\begin{array}{l}\text { Las infraestructuras de transportes y comunicaciones chinas son } \\
\text { modernas. }\end{array}$ & $3,59 *$ & $14,1 \%$ & $42,9 \%$ & $43,0 \%$ \\
\hline $\begin{array}{l}\text { La población china tiene buenos niveles de conocimiento de } \\
\text { idiomas. }\end{array}$ & $2,92 * *$ & $28,9 \%$ & $24,3 \%$ & $46,8 \%$ \\
\hline Los habitantes chinos son muy hospitalarios. & $3,44 *$ & $9,4 \%$ & $30,4 \%$ & $60,2 \%$ \\
\hline $\begin{array}{l}\text { Para un extranjero, resulta fácil y cómodo viajar por libre dentro de } \\
\text { China. }\end{array}$ & $2,29 *$ & $47,7 \%$ & $9,7 \%$ & $42,6 \%$ \\
\hline $\begin{array}{l}\text { Al viajar a China es mejor hacerlo mediante la contratación de } \\
\text { paquetes turísticos. }\end{array}$ & $4,08 *$ & $5,5 \%$ & $71,6 \%$ & $23,0 \%$ \\
\hline $\begin{array}{l}\text { Se dispone de mucha y buena información sobre la oferta turística } \\
\text { china. }\end{array}$ & $2,56 *$ & $50,9 \%$ & $19,2 \%$ & $29,9 \%$ \\
\hline
\end{tabular}

*El test t de la media rechaza la hipótesis nula de no haber diferencia de valor 3 (indiferente) con una alfa de 0,01

**El test t de la media rechaza la hipótesis nula de no haber diferencia de valor 3 (indiferente) con una alfa de 0,05

Fuente: elaboración propia.

Esta hipótesis de relativo desconocimiento de determinados componentes o atributos de la oferta turística china se veía reforzada por las respuestas obtenidas cuando se preguntaba sobre los aspectos en los cuales China destacaba. Como puede observarse en la Cuadro 5, en una escala de 1 a 5, China destacaba, según los entrevistados, por su patrimonio, su cultura claramente diferenciada de la española, su patrimonio natural, las posibilidades de realizar compras, la modernidad de algunas de sus ciudades y su gastronomía. En este conjunto de atributos parecían existir muy pocas dudas entre la población española. Sin embargo, el grado de duda y/o desconocimiento era mucho mayor, superando en todo caso el $40 \%$, en aspectos relativos a oferta cultural, calidad del servicio, calidad del alojamiento, limpieza e higiene, actividades de aventura, oferta de ocio nocturno y casinos. Más del $43 \%$ de los entrevistados no parecían tener claro si China era o no un destino de turismo sexual. En particular, 
merecen atención las moderadas puntuaciones obtenidas en aspectos de capital importancia para el atractivo y competitividad de un destino tales como calidad del servicio y del alojamiento y, especialmente, la percepción de que China era un destino con pobres estándares de limpieza e higiene.

CUADRO 5 - ELEMENTOS EN LOS QUE DESTACA CHINA.

\begin{tabular}{|c|c|c|c|c|}
\hline En mi opinión, China destaca por... & & 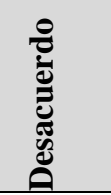 & $\frac{8}{0}$ & 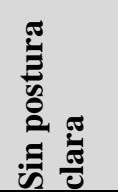 \\
\hline ...el patrimonio histórico. & $4,37 *$ & $2,9 \%$ & $87,8 \%$ & $9,3 \%$ \\
\hline ...la diferencia cultural entre China y España. & $4,27 *$ & $3,5 \%$ & $85,1 \%$ & $11,4 \%$ \\
\hline ...el patrimonio natural (paisaje y entorno). & $4,09 *$ & $6,7 \%$ & $80,2 \%$ & $13,2 \%$ \\
\hline ...la oferta comercial, posibilidad de realizar compras. & $3,94 *$ & $10,6 \%$ & $68,7 \%$ & $20,7 \%$ \\
\hline ...la modernidad de algunas de sus ciudades. & $3,92 *$ & $9,3 \%$ & $65,9 \%$ & $24,8 \%$ \\
\hline ...la gastronomía. & $3,73 *$ & $16,7 \%$ & $65,9 \%$ & $17,4 \%$ \\
\hline ...los grandes eventos (festivales, competiciones, etc.). & $3,42 *$ & $18,2 \%$ & $43,4 \%$ & $38,5 \%$ \\
\hline ...la oferta cultural (arte, ópera, teatro, etc.) & $3,33 *$ & $19,5 \%$ & $38,6 \%$ & $42,0 \%$ \\
\hline ...la calidad del servicio. & 3,06 & $20,7 \%$ & $24,4 \%$ & $54,9 \%$ \\
\hline ...la calidad del alojamiento. & 3,04 & $19,5 \%$ & $20,8 \%$ & $59,7 \%$ \\
\hline ...el turismo sexual. & $2,90 * *$ & $29,6 \%$ & $26,7 \%$ & $43,7 \%$ \\
\hline ...la oferta de ocio nocturno. & $2,81 *$ & $28,3 \%$ & $17,4 \%$ & $54,3 \%$ \\
\hline ...los casinos de juego. & $2,77 *$ & $30,1 \%$ & $17,9 \%$ & $52,0 \%$ \\
\hline ...las actividades de aventura. & $2,61 *$ & $37,3 \%$ & $15,3 \%$ & $47,4 \%$ \\
\hline ...la limpieza e higiene. & $2,48 *$ & $44,8 \%$ & $14,1 \%$ & $41,1 \%$ \\
\hline ... la oferta de sol y playa. & $1,91 *$ & $65,6 \%$ & $5,2 \%$ & $29,3 \%$ \\
\hline
\end{tabular}

*El test $t$ de la media rechaza la hipótesis nula de no haber diferencia de valor 3 (indiferente) con una alfa de 0,01 .

**El test t de la media rechaza la hipótesis nula de no haber diferencia de valor 3 (indiferente) con una alfa de 0,05 .

Fuente: elaboración propia.

\subsection{INHIBIDORES DEL VIAJE A CHINA}

Vemos por los resultados mencionados que China era un destino poco conocido pero atractivo para los encuestados, pero para obtener una imagen más fidedigna del potencial de China como destino turístico era interesante conocer que elementos podrían hacer desistir a los potenciales turistas de realizar el viaje. 


\begin{tabular}{|c|c|c|c|c|}
\hline & 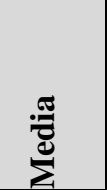 & 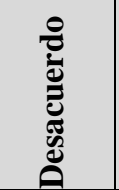 & 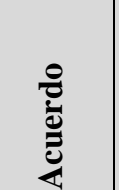 & 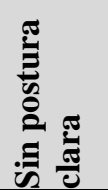 \\
\hline El coste del viaje hasta China es elevado. & $4,40 *$ & $3,3 \%$ & $83,3 \%$ & $13,5 \%$ \\
\hline $\begin{array}{l}\text { El crecimiento económico chino ha hecho gran daño al medio } \\
\text { ambiente del país. }\end{array}$ & $4,22 *$ & $4,2 \%$ & $75,4 \%$ & $20,4 \%$ \\
\hline $\begin{array}{l}\text { La distancia física entre España y China no dificulta en gran medida } \\
\text { los viajes a ese país. }\end{array}$ & $2,53 *$ & $59,9 \%$ & $27,6 \%$ & $12,5 \%$ \\
\hline $\begin{array}{l}\text { Las condiciones socio-políticas chinas no condicionan la decisión de } \\
\text { viajar a China. }\end{array}$ & $2,85^{*}$ & $42,5 \%$ & $32,2 \%$ & $25,3 \%$ \\
\hline $\begin{array}{l}\text { Me desagrada que para ver los sitios atractivos de China haya que } \\
\text { coger tantos medios de transporte. }\end{array}$ & $3,66^{*}$ & $8,0 \%$ & $43,3 \%$ & $48,7 \%$ \\
\hline China es un lugar seguro para los turistas. & 2,97 & $29,9 \%$ & $28,0 \%$ & $42,1 \%$ \\
\hline
\end{tabular}

*El test t de la media rechaza la hipótesis nula de no haber diferencia de valor 3 (indiferente) con una alfa de 0,01 .

Fuente: elaboración propia.

Los resultados obtenidos (Cuadro 6) parecen indicar claramente que el mayor inhibidor del potencial viaje a China era el elevado coste del viaje, seguido de la distancia física entre ambos países (que, a la vez, incide sobre el coste del viaje tanto en términos económicos, como de tiempo). La percepción de que el desarrollo económico chino se ha hecho a costa de una gran degradación medioambiental podría actuar también como un inhibidor, dados los crecientes niveles de preocupación por cuestiones medioambientales en el conjunto de la población de Europa Occidental. También parecían tener un cierto peso relativo como inhibidores las condiciones socio-políticas chinas y el hecho de tener que realizar desplazamientos importantes una vez que se está en China si se quieren ver los atractivos turísticos más importantes. Por último, el hecho de no tener muy claro si China era un lugar seguro para los turistas.

\section{CONCLUSIONES}

China era percibida por los españoles como la gran potencia del futuro, un país económicamente desarrollado, con un gran patrimonio histórico y cultural, pero que presta muy poca atención a la preservación del medio ambiente. China era una gran desconocida pero, al mismo tiempo resulta atractiva. Existía un desconocimiento bastante generalizado de China como destino turístico entre los españoles, aunque 
reconocían que es un país con abundantes recursos turísticos y con un patrimonio histórico, cultural y natural importante. Los españoles tenían muchas dudas respecto a determinados componentes de la oferta turística que tienen una importancia fundamental de cara a la competitividad internacional de un destino (calidad, seguridad del destino, infraestructuras de transporte, hospitalidad de la gente, limpieza e higiene, entre otros).

El potencial emisor del mercado español parecía elevado, si se valora en términos de intención de visitar el país. El gran potencial estaba en el turismo cultural y urbano, aunque el turismo de naturaleza tiene posibilidades importantes. Sin embargo, determinados aspectos actúan como inhibidores del viaje a China. El principal es el coste en términos monetarios y de tiempo. Pero, a continuación, aparecían otros elementos que pueden desanimar la visita como cuestiones relacionadas con la situación política y las condiciones de seguridad para los turistas.

Los resultados parecen indicar que uno de los aspectos que incidían negativamente en la imagen turística de China eran las cuestiones medioambientales y la conveniencia, por tanto, de introducir políticas más sostenibles en el futuro que incrementarían sensiblemente su atractivo como destino turístico.

Los datos sugieren la conveniencia de incrementar y/o mejorar las acciones de comunicación en todo lo relativo a China como destino turístico: recursos turísticos, actividades que pueden realizarse, entre otras, pero, sobre todo, en cuestiones que ayuden a reducir el grado de incertidumbre que el potencial visitante puede tener ante la perspectiva de un viaje tan largo: calidad de la oferta de alojamiento, calidad del servicio, hospitalidad de los residentes, seguridad del destino y libertad de movimientos, calidad de los servicios de asistencia sanitaria (en el caso de que se requieran), conocimiento de idiomas por parte del personal de contacto con el cliente, entre otras. Ante determinados inhibidores, como coste y distancia, poco puede hacerse, pero ante otros, derivados de un relativo desconocimiento de la realidad, las acciones de comunicación pueden ser muy efectivas. En conjunto, si se toman las medidas adecuadas, las perspectivas para el desarrollo de los flujos turísticos hacia China eran positivas. 


\section{REFERENCIAS}

BALOGLU, S.; MANGALOGLU, M. Tourism destination images of Turkey, Egypt, Greece, and Italy as perceived by US-based tour operators and travel agents. Tourism Management, v. 22, n. 1, p. 1-9, 2001.

BIGNÉ, J. E.; SÁNCHEZ, M. I.; SÁNCHEZ, J. Tourism image, evaluation variables and after purchase behaviour: inter-relationship. Tourism Management, v. 22, n. 6, p. 607-616, 2001.

BIGNÉ, J. E.; SÁNCHEZ, M. I.; SANZ, S. The functional-psychological continuum in the cognitive image of a destination: A confirmatory analysis. Tourism Management, v. 30, n. 5, p. 715-723, 2009.

CHEN, Y. B. A study on the impact of Korean Wave on Korea's tourism image. The Journal of Tourism Policy, v. 12, n. 1, p. 63-78, 2005.

CHINA NATIONAL TOURIST OFFICE (CNTO). Disponible en: <http://www.cnto.org/chinastats.asp>. Acceso en: 27/12/2012.

CHOI, J. G.; TKACHENKO, T.; SIL, S. On the destination image of Korea by Russian tourists. Tourism Management, v. 32, n. 1, p. 193-194, 2011.

CHOI, W. M.; CHAN, A.; WU, J. A qualitative and quantitative assessment of Hong Kong's image as a tourist destination. Tourism Management, v. 20, n. 3, p. 361-365, 1999.

CROMPTON, J. L. An assessment of the image of Mexico as a vacation destination and the influence of geographical location upon that image. Journal of Travel Research, v. 17, n. 4, p. 18-23, 1979.

ECHTNER, C. M.; RITCHIE, J. R. The meaning and measurement of destination image. Journal of Tourism Studies, v. 2, n. 2, p. 2-12, 1991.

The measurement of destination image: An empirical assessment. Journal of Travel Research, v. 31, n. 4, p. 3-13, 1993.

GAllarZA, M. G.; SAURA, I. G.; GARCIA, H. C. Destination image towards a conceptual framework. Annals of Tourism Research, v. 29, n. 1, p. 56-78, 2002.

GIBSON, H. J.; QI, C. X.; ZHANG, J. J. Destination image and intent to visit China and the 2008 Beijing Olympic Games. Journal of Sport Management, v. 22, n. 4, p. 427-450, 2008.

GREEN, B. C.; LIM, S. Y.; SEO, W. J.; SUNG, Y. Effects of cultural exposure through pre-event media. Journal of Sport and Tourism, v. 15, n. 1, p. 89-102, 2010. 
GUO, Y.; KIM, S. S.; TIMOTHY, D. J. Development characteristics and implications of Mainland Chinese outbound tourism. Asia Pacific Journal of Tourism Research, v. 12, n. 4, p. 313-332, 2007.

HESLOP, L. A.; NADEAU, J.; O'REILLY, N. China and the Olympics: Views of insiders and outsiders. International Marketing Review, v. 27, n. 4, p. 404-433, 2010.

HUANG, S.; HSU, C. H. C. Effects of travel motivation, past experience, perceived constraint, and attitude on revisit intention. Journal of Travel Research, v. 48, n. 1, p. 29-44, 2009.

HUNT, J. D. Image as a factor in tourism development. Journal of Travel Research, v. 13, n. 1, p. 1-7, 1975.

INSTITUTO DE TURISMO DE ESPAÑA. China. Estudio de demanda 2008. Estudios de mercados emisores. Madrid: Instituto de Turismo de España (Turespaña), 2009.

INSTITUTO NACIONAL DE ESTADÍSTICA. Disponible en: <http://www.ine.es/> . Acceso en: 27/12/2012.

JEFFREY, D.; XIE, Y. The UK market for tourism in China. Annals of Tourism Research, v. 22, n. 4, p. 857-876, 1995.

JENKINS, O. H. Understanding and measuring tourist destination images. International Journal of Tourism Research, v. 1, n. 1, p. 1-15, 1999.

KIM, S. S.; MORRISON, A. M. Change of images of South Korea among foreign tourists after the 2002 FIFA world cup. Tourism Management, v. 26, n. 2, p. 233-247, 2005.

KIM, S. S.; GUO, Y.; AGRUSA, J. Preference and positioning analyses of overseas destinations by Mainland Chinese outbound pleasure tourists. Journal of Travel Research, v. 44, n. 2, p. 212-220, 2005.

LAW, R.; CHEUNGM S. The perceived destination image of Hong Kong as revealed in the travel blogs of mainland Chinese tourists. International Journal of Hospitality and Tourism Administration, v. 11, n. 4, p. 303-327, 2010.

LEE, C-K.; LEE, Y-K.; LEE, B. Korea's destination image formed by the 2002 world cup. Annals of Tourism Research, v. 32, n. 4, p. 839-858, 2005.

LEE, R.; LOCKSHIN, L. Halo effects of tourists' destination image on domestic product perceptions. Australasian Marketing Journal, v. 19, n. 1, p. 7-13, 2011.

LEE, T. H. A development of Korea destination image measurement scale. Korean Journal of Tourism Research, v. 20, n. 2, p. 80-95, 1997. 
LEUNG, D.; LAW, R.; LEE, H. A. The perceived destination image of Hong Kong on Ctrip.com. International Journal of Tourism Research, v. 13, n. 2, p. 124-140, 2011.

LI, F. M. S. Culture as a major determinant on tourism development on China. Current Issues in Tourism, v. 11, n. 6, p. 492-513, 2008.

LI, X.; VOGELSONG, H. Comparing methods of measuring image change: A case study of a small-scale community festival. Tourism Analysis, v. 10, n. 4, p. 349-360, 2006.

LI, X.; PAN, B.; ZHANG, L. G.; SMITH, W. W. The effect of online information search on image development: Insights from a mixed-methods study. Journal of Travel Research, v. 48, n. 1, p. 45-57, 2009.

MCCARTNEY, G.; BUTLER, R.; BENNETT, M. A strategic use of the communication mix in the destination image-formation process. Journal of Travel Research, v. 47, n. 2, p. 183-196, 2008.

MCCLINCHEY, K. A. Rural images tourism and sustainability: Perceptions of rural accommodation operators and their visitors in Waterloo-Wellington Region. Ontario: Wilfrid Laurier University, 1999.

NATIONAL TOURISM ADMINISTRATION OF THE PEOPLE'S REPUBLIC OF CHINA (CNTA). Disponible en: <http://en.cnta.gov.cn/>. Acceso en: 27/12/2012.

$\mathrm{NOH}$, J. H. Factors influencing American's intentions to vacation in South Korea. Korean Journal of Tourism Research, v. 22, n. 3, p. 163-182, 2007.

PAN, B.; LI, X. The long tail of destination image and online marketing. Annals of Tourism Research, v. 38, n. 1, p. 132-152, 2011.

PIKE, S. Destination image analysis: A review of 142 papers from 1973 to 2000. Tourism Management, v. 23, n. 5, p. 541-549, 2002.

SANTESMASES, M. DYANE Versión 4. Diseño y Análisis de Encuestas en Investigación Social y de Mercados. Madrid: Editorial Pirámide, 2009.

SEGUÍ, M.; CAPELLÀ, J. E. Spanish package holiday tourism to China: Spatial patterns and tourist attractions. Tourism Geographies, v. 8, n. 3, p. 233-252, 2006.

SELBY, M.; MORGAN, N. J. Reconstruing place image: A case study of its role in destination market research. Tourism Management, v. 17, n. 4, p. 287-294, 1996.

SHANI, A.; CHEN, P-J.; WANG, Y.; HUA, N. Testing the impact of a promotional video on destination image change: Application of China as a tourism destination. International Journal of Tourism Research, v. 12, n. 2, p. 116-133, 2010. 
SPARKS, B.; PAN, G. W. Chinese outbound tourists: Understanding their attitudes, constraints and use of information sources. Tourism Management, v. 30, n. 4, p. 483494, 2009.

STEPCHENKOVA, S.; MORRISON, A. M. Russia's destination image among American pleasure travelers: Revisiting Echtner and Ritchie. Tourism Management, v. 29, n. 3, p. 548-560, 2008.

SU, M. M.; WALL, G. The Qinghai-Tibet railway and Tibetan tourism: Travelers' perspectives. Tourism Management, v. 30, n. 5, p. 650-657, 2009.

TASCI, A. D. A.; GARTNER, W. C.; CAVUSGIL, S. T. Conceptualization and operationalization of destination image. Journal of Hospitality and Tourism Research, v. 31, n. 2, p. 194-223, 2007.

TSANG, N. K. F.; HSU, C. H. C. Thirty years of research on tourism and hospitality management in China: A review an analysis of journal publications. International Journal of Hospitality Management, v. 30, n. 4, p. 886-896, 2011.

UNWTO. Tourism Highlights 2010 Edition. Madrid: World Tourism Organization, 2011. Disponible en: <http://www.unwto.org/facts/menu.html>. Acceso en: 27/12/2012.

VAN DER VEEN, R.; SONG, H. Exploratory study of the measurement scales for the perceived image and advertising effectiveness of celebrity endorsers in a tourism context. Journal of Travel and Tourism Marketing, v. 27, n. 5, p. 460-473, 2010.

YANG, C. H.; LIN, H. L.; HAN, C. C. Analysis of international tourist arrivals in China: The role of World Heritage Sites. Tourism Management, v. 31, n. 6, p. 827837, 2010.

YANG, J.; HE, J.; GU, Y. The implicit measurement of destination image: The application of Implicit Association Tests. Tourism Management, v. 33, n. 1, p. 50-52, 2012.

ZHANG, H. Q.; CHOW, I. Application of importance-performance model in tour guides' performance: evidence from Mainland Chinese outbound visitors in Hong Kong. Tourism Management, v. 25, n. 1, p. 81-91, 2004.

Recebido em: 09-02-2014.

Aprovado em: 07-03-2014. 